\title{
Total Internal Reflection-Based High Efficiency Grating Design for a Metal-Free Polarizing Filter Applications Using Hybrid Optimization Procedure
}

\author{
ChaBum Lee \\ Department of Mechanical Engineering, University of South Carolina, 300 Main Street, Columbia, SC 29208, USA \\ Correspondence should be addressed to ChaBum Lee; chabum@mailbox.sc.edu
}

Received 28 October 2013; Revised 23 December 2013; Accepted 31 December 2013; Published 10 February 2014

Academic Editor: José Luís Santos

Copyright (c) 2014 ChaBum Lee. This is an open access article distributed under the Creative Commons Attribution License, which permits unrestricted use, distribution, and reproduction in any medium, provided the original work is properly cited.

\begin{abstract}
This paper presents a fast and rigorous design method for grating-based metal-free polarizing filter applications using two-step hybrid optimization techniques. Grating structures utilizing the total internal reflection in a lamellar configuration were used to achieve metal-free solution, which is a key technology in the chirped pulse amplification for high power laser system. Here two polarizing filters were designed: polarization sensitive and polarization insensitive. Those polarization performances were characterized by the rigorous coupled-wave analysis (RCWA), and the design parameters of grating structures, pitch, depth, and filling factor were optimized by two-step hybrid optimization procedure because the diffraction characteristics of grating-based polarizing filters are highly sensitive to small changes in design parameters. The Taguchi method is incorporated into selection process in the genetic algorithm, which indicates that the Taguchi method optimizes the design parameters in a coarse manner, and then, coarsely optimized parameters are finely optimized using the genetic algorithm. Therefore the proposed method could solve global numerical optimization problems with continuous variables. The proposed two-step hybrid optimization algorithm could effectively optimize the grating structures for the purpose of polarization filter applications, and the optimized grating structures could selectively filter the incident light up to $99.8 \%$ as to TE or TM waves.
\end{abstract}

\section{Introduction}

Diffraction gratings play an important role in many optical applications, including optical telecommunications components, spectroscopy, and the chirped pulse amplification (CPA) for a high power laser system [1-4]. Especially, diffraction gratings utilizing total internal reflection (TIR) within a metal-free groove are an environmentally friendly integrated optical element expected to achieve a high performance at a specific retrodiffracted order [1-4]. Absorption of the incident light at the metallic surface diminishes diffraction efficiency (DE) to $75-95 \%$, depending on the material and wavelength of the incident light, which can result in the severe damages on the grating facets because this absorbed energy turns into heat [3]. Therefore, the TIR-based dielectric gratings can be available to provide a metal-free solution and a high performance. Thanks to current manufacturing technology such as E-Beam lithography, photolithography, and holography, fabrication of grating structures can be achieved up to nanometer accuracy, which means that fabrication error can be assumed to be negligible. However, diffraction characteristics of the TIR-based grating structures are highly sensitive to those geometric properties, pitch, filling factor, and depth, that the proper design methods to be able to solve global numerical optimization problems with continuous variables have been becoming issues for many optical applications [5-8]. Here the optical analysis method was combined with the hybrid optimization methods to evaluate the performance of the TIR-based grating structure and find out the optimal set of design parameters at the same time.

Scalar diffraction theory is one of the widely used methods for the design and analysis of grating structures [7-10]. The Fresnel and Fraunhofer diffraction integrals are 
commonly adopted, and these signals are generally evaluated with the fast Fourier transform (FFT). Although this approach is relatively simple and shows a good agreement with experimental results to some extent, its applicability is somehow limited due to diffraction limit. When the grating structure becomes so small that it is comparable to or less than the wavelength, the simplified approximations made in the scalar method are not valid and the polarization nature of the light cannot be ignored in this regime. Therefore, a more accurate fully electromagnetic analysis is required. The rigorous coupled-wave analysis (RCWA) provides a means to solve these problems, and it has been recently used to characterize the diffraction phenomenon of the grating structures $[10,11]$.

In general, diffraction gratings with a lamellar configuration consist of a periodically varying boundary of the period $P$ between two different media with refractive indices $n_{0}$ and $n_{i}$ as illustrated in Figure 1, where filling factor $F$, depth $D$, wavelength $\lambda$ and incident angle $\theta_{i}$ and $\theta_{m}$ the $m$ th order diffraction angle, respectively. The TIR-based grating equation may be expressed as $[3,4]$

$$
\begin{aligned}
& n_{i} \sin \left(\theta_{i}\right)+n_{0} \sin \left(\theta_{m}\right)+m \frac{\lambda}{P}=0, \\
& n_{0}<\frac{\lambda}{2 P}<n_{i} .
\end{aligned}
$$

Whereas the angular and spectral characteristics of gratings solely depend on (1), DE is dependent on the surface profile of gratings, which is calculated by the rigorous coupled-wave analysis (RCWA) [7-11]. In accordance with the TIR-based grating condition, it might be a big obstacle to obtain the optimum grating parameters, $P, F$, and $D$. For this case, a stochastic approach can be employed, but it costs time and a question remains if a solution is a global minimum or not [12-18]. In recent years, hybrid optimization techniques are provided to improve the search performance and reduce the computational complexity of design [12,13]. Anthony employed the robustness issues and genetic algorithm (GA) to produce optimal solutions for two-dimensional structural applications [14]. Fang et al. used the hybrid genetic algorithm for a zoom optics application [15]. Huang et al. introduced the design procedure of thin-film filters using the simulated annealing algorithm [18]. However, there was little work on the optimization of the TIR-based gratings. Lee et al. demonstrated the parametric optimization to grating structures using the Taguchi method [4]. This approach provided the design concept for the TIR gratings but did not reach the global minimum, which means that its performance is still in short when it takes TIR into consideration. Here twostep optimization procedures using hybrid optimization techniques, the hybrid Taguchi-GA (HTGA), were used to solve multiobjective global numerical optimization problems with continuous variables through a smaller population of individual results in a solution, and this approach was applied to design of the grating-based metal-free polarizing filter applications. The two TIR-based gratings with the polarizationsensitive and -insensitive performance were calculated by RCWA and those geometric parameters, pitch, depth, and

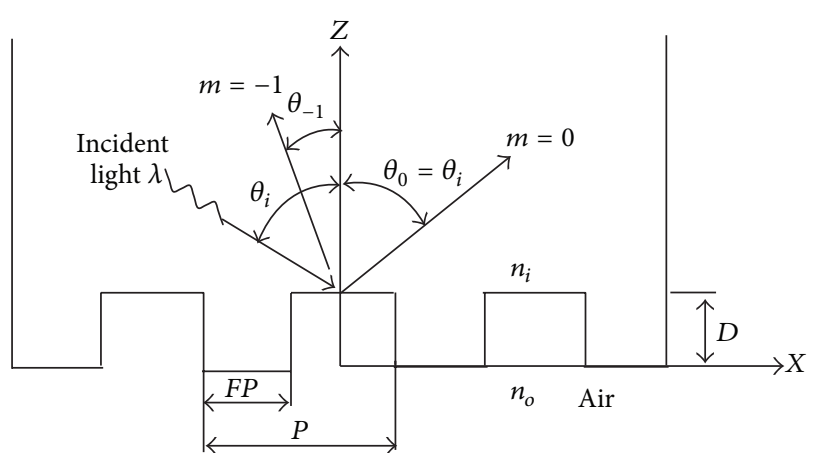

FIgURE 1: Schematic of the TIR gratings in a lamellar configuration.

filling factor, were optimized by HTGA, respectively. Also, changes in DE with respect to each parameter error were discussed.

\section{Theories}

2.1. Rigorous Coupled-Wave Analysis. RCWA is one of direct methods to solve the Maxwell's equations based on a state space representation without any assumptions $[10,11]$. This method has been widely used for analysis of many diffractive optical elements. When an electromagnetic wave is incident upon the grating structures at a certain angle, part of the wave is reflected back and the rest is transmitted to the grating structures as a result of backward and forward diffraction as seen in Figure 1. The coupled-wave equations can be solved by matching the tangential electric and magnetic field components at each boundary layer. As a result, the $i$ th reflection efficiency and transmission efficiencies with respect to polarization direction, TE (electric field perpendicular to the incident plane), and TM (magnetic field perpendicular to the incident plane) can be calculated by (2), respectively,

$$
\mathrm{DE}_{i}=R_{i} R_{i}^{*} \operatorname{Re}\left(\frac{k_{1 i}}{k_{0} n_{1} \cos \theta_{i}}\right),
$$

where $R_{i}$ is the $i$ th reflectance and $k$ is a wavevector.

2.2. Taguchi Method. The following optimization problem is considered:

$$
\begin{array}{ll}
\text { minimize } & F(X), \\
\text { subject to } & \mathrm{LB} \leq X \leq \mathrm{UB},
\end{array}
$$

where $X=\left(X_{1}, X_{2}, \ldots, X_{i}, \ldots, X_{N}\right)$ is a variable vector in real space, $N, F(X)$ is a fitness function, $\mathrm{LB}$ is a lower boundary, and UB is an upper boundary defined in the feasible solution space. The Taguchi method, a robust design approach, uses many ideas from statistical experimental design for evaluating and implementing improvements in products, processes, and equipment $[16,17]$. The fundamental principle is to improve the quality of a product by minimizing the effect of the causes of variation without eliminating the 


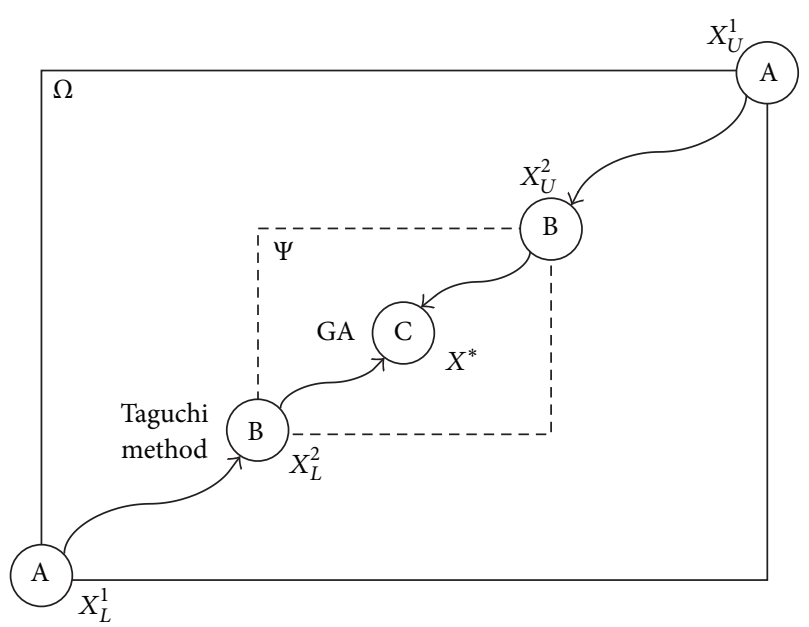

FIgURE 2: Optimization procedure of HTGA.

causes. In this method, the two major tools are used: signalto-noise (SNR) and analysis of variance (ANOVA):

$$
\mathrm{SNR}=-10 \log \left[\frac{1}{N} \sum_{i=1}^{N}\left(\frac{1}{F\left(X_{i}\right)^{2}}\right)\right]
$$

where $N$ is the number of experiments in the orthogonal arrays. The former is used to measure is quality and the later are used to analyze the difference between group means and variation in orthogonal arrays at the same time. Through those tools, the optimal set of values can be obtained at the end.

2.3. Genetic Algorithm. The genetic algorithm is a class of adaptive stochastic optimization algorithms involving search and optimization $[12,13]$. The fundamental principle is to solve both constrained and unconstrained optimization problems based on a natural process that mimics biological evolution. The first step is to mutate, or randomly vary, a given collection of sample programs by

$$
\begin{gathered}
X_{i}=\mathrm{LB}_{i}+\beta\left(\mathrm{UB}_{i}-\mathrm{LB}_{i}\right), \\
X_{i}^{\prime}=(1-\beta) X_{i}+\beta X_{j}, \\
X_{j}=\beta X_{i}+(1-\beta) X_{j},
\end{gathered}
$$

where $\beta$ is the crossover fraction in reproduction and mutation processes, respectively. $X_{i}$ and $X_{j}$ are randomly selected for mutation, and the resulting offspring becomes $X_{i}^{\prime}$. The second step is a selection step, which is often done through measuring against a fitness function. The process is repeated and a population of individual solutions is updated until a suitable solution is found.

\section{Optimization Procedure}

The proposed HTGA optimization procedure was illustrated in Figure 2; $X=\langle P, F, D\rangle$ can exist only in all sets of admissible space $\Omega$ and the initial boundary A, quasioptimized boundary B, and globally optimized point C. A coarse estimation from A to B was performed by the Taguchi method and a fine estimation from $\mathrm{B}$ to $\mathrm{C}$ was by GA, respectively. Based on the analysis results of $S / N$ ratio and ANOVA of each parameter, the Taguchi method narrows down all sets of admissible space $\Omega$ to the quasi-admissible space $\Psi$, which provides the narrowed-down search region for GA. The three-level standard orthogonal arrays, $L_{18}$, were employed [4]. This two-step procedure allows saving the computation time to find the global minimum. To employ $\mathrm{GA}$, the fitness function can be expressed as $[13,14]$

$$
F(X)=\left[f(X)-f\left(X^{*}\right)\right]^{2}, \quad \forall X=\langle P, F, D\rangle,
$$

where $f\left(X^{*}\right)$ is a target value and $f(X)$ is a current value. $f(X)$ is a function of DE at the -1 st order. Population of each parameter was set to 15 and crossover fractions were set to 0.8 and 1.0 for reproduction and mutation, respectively. Here RCWA code was implemented into HTGA. In the RCWA calculation, the refractive index of substrate $\left(n_{i}=1.65\right.$, heavy flinted glass), the wavelength $(\lambda=1.068 \mu \mathrm{m}$, commonly used in CPA system), and the incident angle of light $\left(\theta_{i}=45^{\circ}\right)$ for the TIR condition were assumed. DE is defined as the fraction of incident monochromatic light diffracted into a specific order. It is assumed that the sum of DE at all harmonic orders keeps $100 \%$, which means lossless. Also, $41(-20 \sim+20)$ space-harmonic components were assumed to be convergent for the numerical calculation. The parameter variation due to thermal effect as a noise factor is so small that it was considered that it does not have an effect on optimization results: glass, $\mathrm{d}(P, F, D) / \mathrm{d} T \sim 10^{-6}(\mu \mathrm{m}) / \mathrm{K}, \mathrm{d} n / \mathrm{d} T \sim 10^{-6} / \mathrm{K}$, $\mathrm{d} n / \mathrm{d} \lambda \sim 10^{-5} / \mathrm{nm}[19]$. The all calculation and optimization procedures were performed in Matlab [18] environment with a personal computer (CPU $1.7 \mathrm{GHz}$, RAM $1 \mathrm{G}$ byte).

\section{Results}

4.1. Polarizing-Sensitive Grating Structures. The hybrid optimization procedure was performed to achieve polarizingsensitive performance from the TIR-based grating structures, and each of the design parameters was carefully chosen. The pitch parameter was set from (1), and filling factor and depth were set in the whole space, $\Omega=\{X(P, F, D) \mid$ $0.330<P[\mu \mathrm{m}]<0.534,0.1<F<0.9,0.1<$ $D[\mu \mathrm{m}]<1.0\}$. The effective set of parameters was coarsely optimized by the Taguchi method [4]. Based on the analysis results of $S / N$ ratio and ANOVA of each parameter, set of the design parameters, $X(0.435,0.5,0.85)$, was obtained, and then, the narrowed-down quasi-admissible space $\Psi=$ $\{X(P, F, D) \mid 0.4<P[\mu \mathrm{m}]<0.5,0.3<F<0.6,0.8<$ $D[\mu \mathrm{m}]<1.0\}$ was subdivided for GA, respectively. HTGA for polarization-sensitive grating design found the optimal set $X(0.46,0.41,0.89)$ within the quasi-admissible space $\Psi$; $\mathrm{DE}_{-1, \mathrm{TE}}=99.8 \%$ and $\mathrm{DE}_{-1, \mathrm{TM}}=0.0 \%$ at $\lambda=1.068 \mu \mathrm{m}$. As seen in Figure 3, the convergences were made at the 13 rd generation and no failure was found while running 20 times. Compared to the results of the Taguchi method, the performance of the TIR grating as a polarizing filter increased 


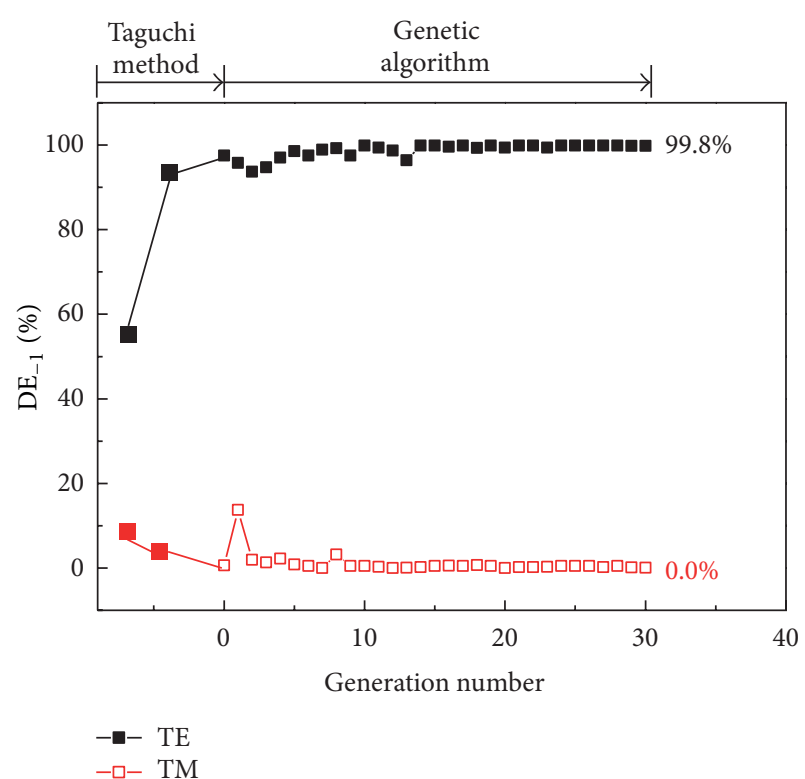

(a)

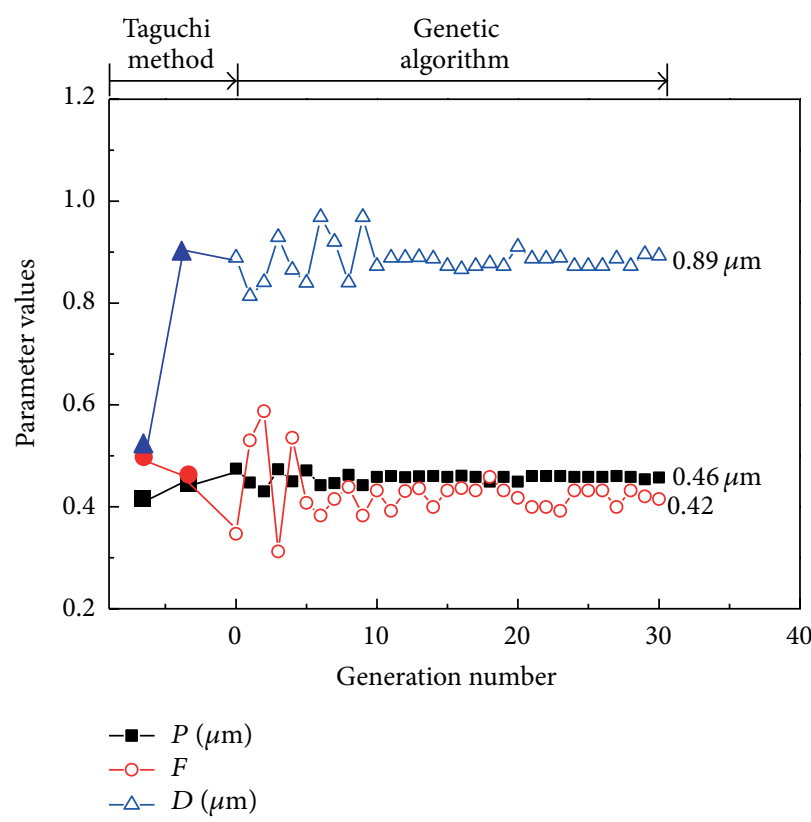

(b)

FIGURE 3: Efficiency (a) and parameter (b) convergence curves of HTGA with respect to generation number for the TIR-based gratings with polarization-sensitive performance.

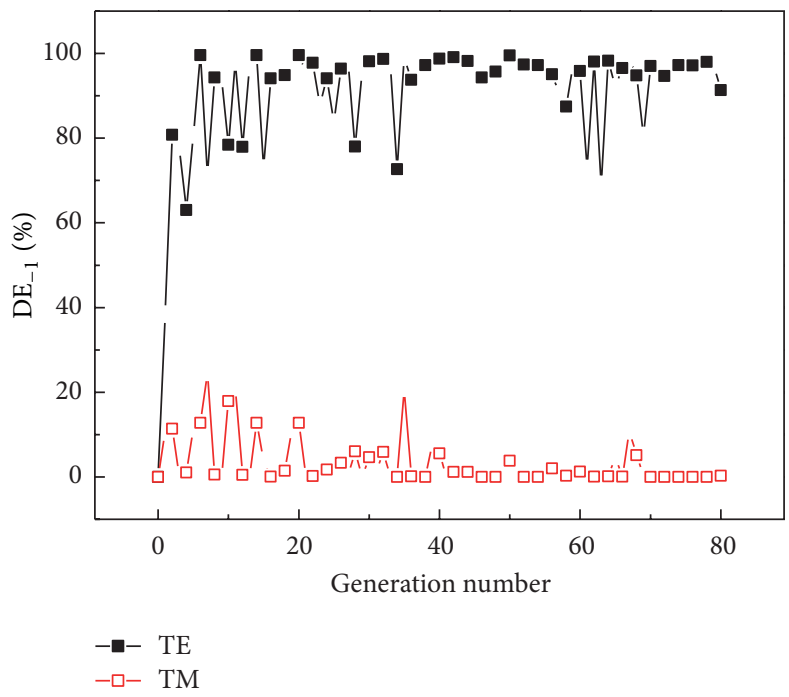

(a)

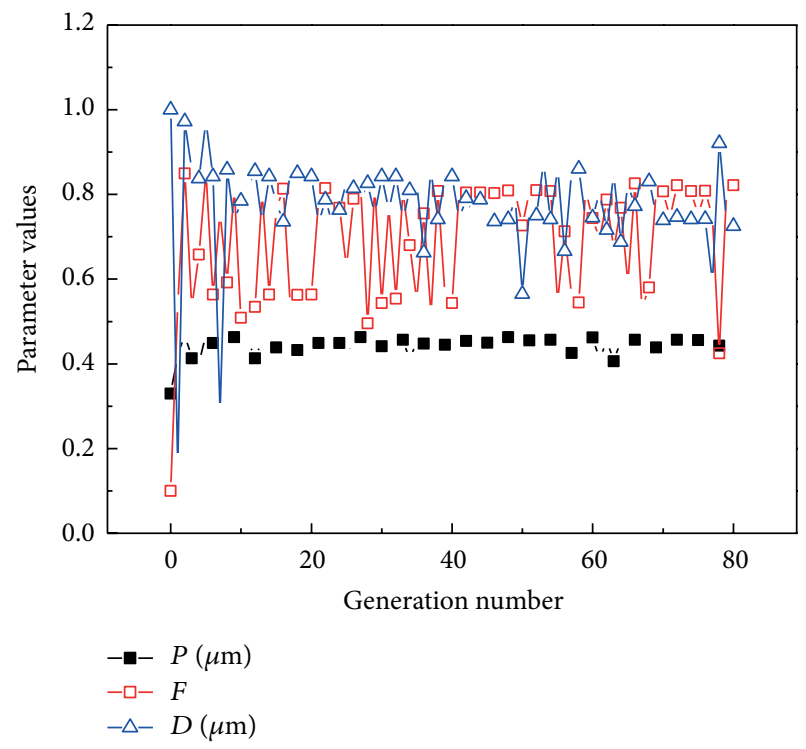

(b)

FIGURE 4: Efficiency (a) and parameter (b) convergence curves of a pure GA with respect to generation number for the TIR-based gratings with polarization-sensitive performance.

by up to $99.8 \%$ by means of the GA. It was seen that all of the feasible values were less than $1.0 \%$ variation of each value and those DEs were almost equal, which indicates that it reached the global minimum. To verify the performance of HTGA, a pure GA without the Taguchi method was applied to obtain the optimal set of parameter values over the whole space as shown in Figure 4. The DEs tended to be converged, but those values were not sufficient to satisfy a TIR-grating performance even though generation number reached 80 . Also, parameters, $F$ and $D$, significantly fluctuated with generation number, which indicates that it cannot single an optical set of parameters out. As a result, the gratings with those values were not ideally polarization sensitive, even the obtained values were randomly distributed in the whole 


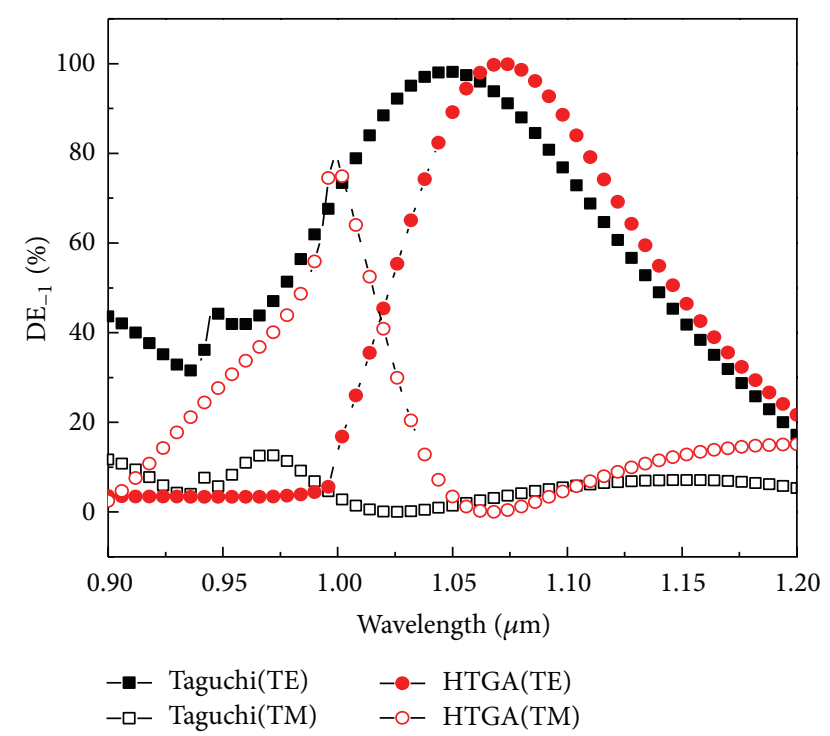

Figure 5: Spectral response curves on the results of the Taguchi method only $X(0.435,0.5,0.85)$ and HTGA $X(0.46,0.41,0.89)$ for the TIR-based grating with polarization-sensitive performance.

space and many failures were found, which is not suitable for solving an optimization problem. It could be seen that HTGA provides a great deal of improvement with regard to its performance and computation time. From the spectral response of the designed gratings shown in Figure 5, the designed gratings provided DE higher than $95 \%$ within $30 \mathrm{~nm}$ bandwidth at a center of $\lambda=1.068 \mu \mathrm{m}$. A peak at $\lambda=1.0 \mu \mathrm{m}$ was thought to result from the resonance of diffracted waves in the gratings. Furthermore, the effect of parameter variation on the DEs was investigated in Figure 6. It was found that the period error among the parameter errors was highly sensitive to DE changes compared to others.

4.2. Polarization-Insensitive Grating Structures. In the same manner with a previous process, the hybrid optimization procedure was performed to achieve polarizing-insensitive performance from the TIR-based grating structures. The pitch parameter was set from (1), and filling factor and depth were set in the whole space, $\Omega=\{X(P, F, D) \mid$ $0.330<P[\mu \mathrm{m}]<0.534,0.1<F<0.9,0.1<$ $D[\mu \mathrm{m}]<1.0\}$. The effective set of parameters was coarsely optimized by the Taguchi method [4]. Based on the analysis results of $S / N$ ratio and ANOVA of each parameter, set of the design parameters, $X(0.45,0.3,0.45)$, was obtained, and then, the narrowed-down quasi-admissible space $\Psi=$ $\{X(P, F, D) \mid 0.4<P[\mu \mathrm{m}]<0.5,0.2<F<$ $0.4,0.4<D[\mu \mathrm{m}]<1.0\}$ was subdivided for GA, respectively. HTGA for polarization-insensitive grating design found the optimal set $X(0.46,0.25,0.49)$ within the quasi-admissible space $\Psi ; \mathrm{DE}_{-1, \mathrm{TE}}=99.9 \%$ and $\mathrm{DE}_{-1, \mathrm{TM}}=99.2 \%$ at $\lambda=1.068 \mu \mathrm{m}$, A small difference in $\mathrm{DE}$ for TE and TM polarization resulted from difference in the refractive index of substrate for polarization direction. As shown in Figure 7, the convergences were made at the 16th generation and no

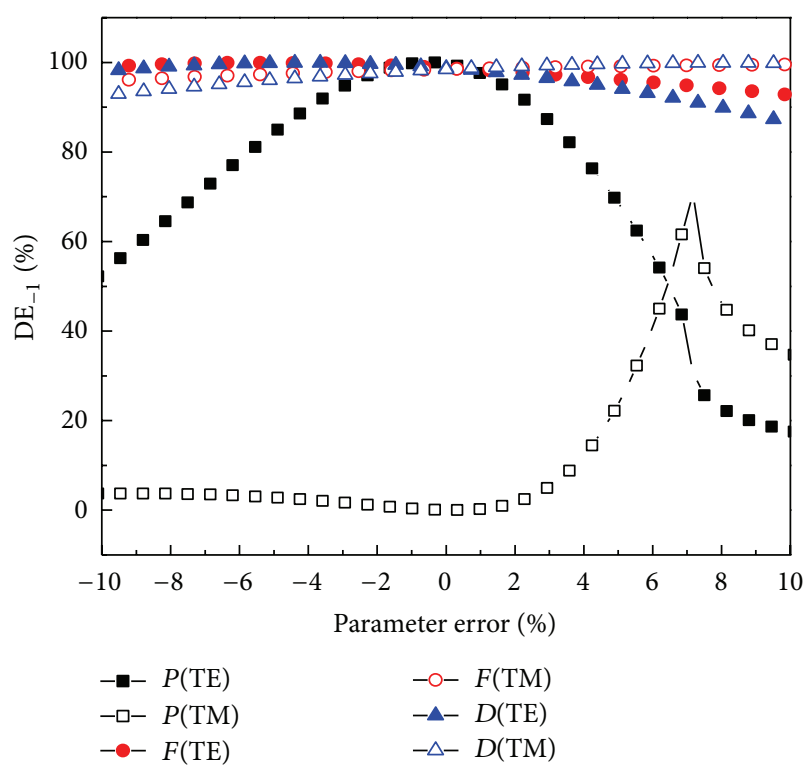

FIGURE 6: Efficiency curves with respect to parameter errors of the TIR-based gratings with polarization-sensitive performance.

failure was found while running 20 times. Compared to the results of the Taguchi method, DE increased by up to $99.9 \%$ and $99.2 \%$ according to its polarization direction by means of the GA. Like the previous analysis, a pure GA without the Taguchi method was applied to obtain the optimal set of parameter values over the whole space. Similarly, DEs were converged towards 95 98\% and parameters showed a similar tendency as DEs. But small differences still existed even though a total generation number was 80 as seen in Figure 8. Thus, it was reconfirmed that HTGA provides a great deal of improvement with regard to its performance and computation time. From the spectral response of the designed gratings shown in Figure 9, the designed gratings provided DE higher than $95 \%$ within $50 \mathrm{~nm}$ bandwidth at a center of $\lambda=1.068 \mu \mathrm{m}$. Furthermore, the effect of parameter variation on the DEs was investigated in Figure 10. Like the previous analysis result, it can be seen that the period error among the parameter errors was highly sensitive to DE changes compared to others and the period parameter has a significant effect on DEs as to TE and TM for the TIR-based grating design.

\section{Conclusion}

We have demonstrated the fast and rigorous multiobjective HTGA optimization procedure, which could solve global numerical optimization problems with continuous variables. This method was applied to optimization of the TIR-based grating structures for polarizing filter applications that the diffraction characteristics are high sensitive to those geometric properties, pitch, filling factor, and depth. RCWA was combined with the hybrid optimization methods to evaluate the performance of the TIR-based grating structure and find out the optimal set of design parameters at the same time. 


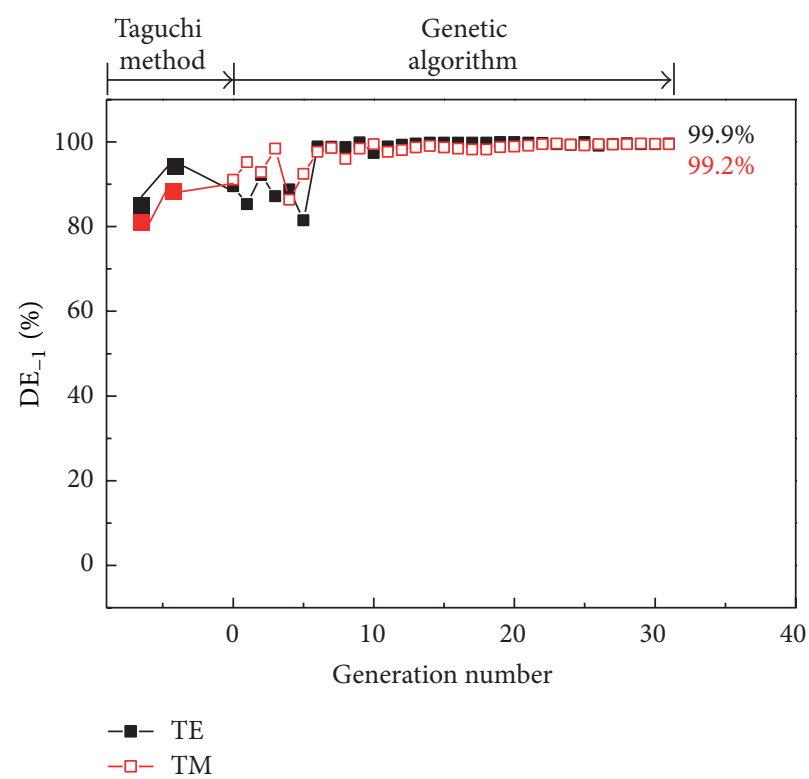

(a)

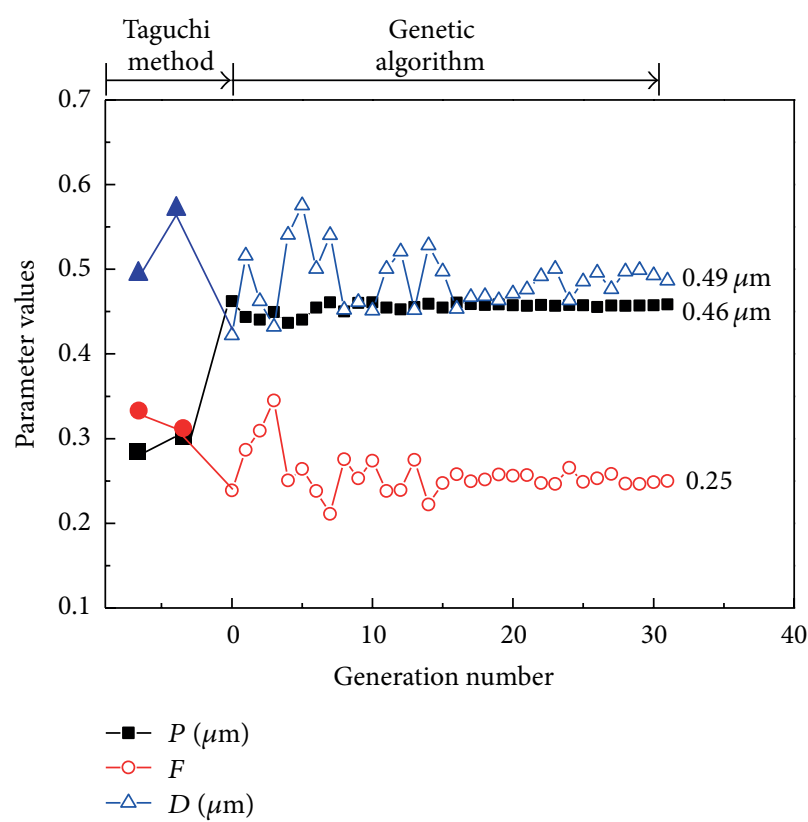

(b)

FIGURE 7: Efficiency (a) and parameter (b) convergence curves of HTGA with respect to generation number for the TIR-based gratings with polarization-insensitive performance.

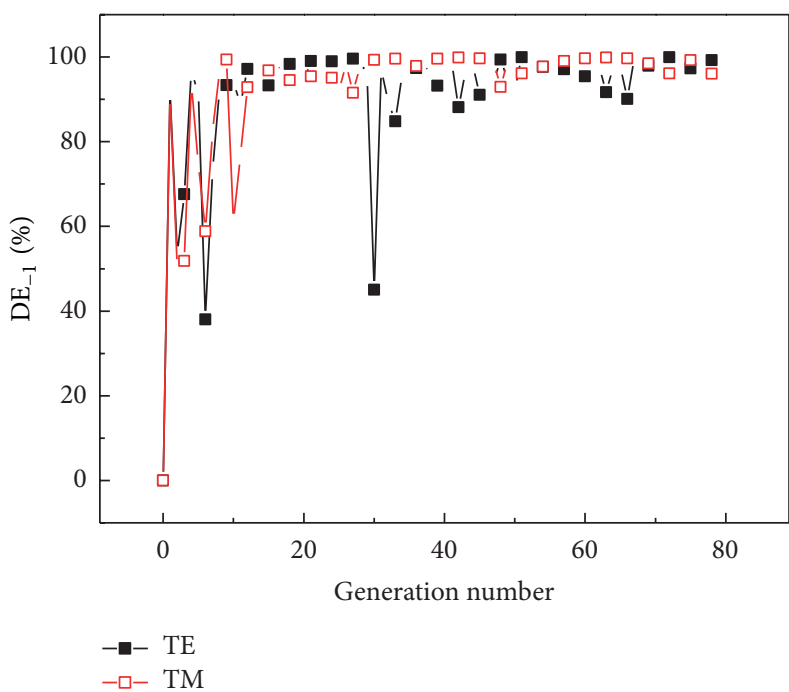

(a)

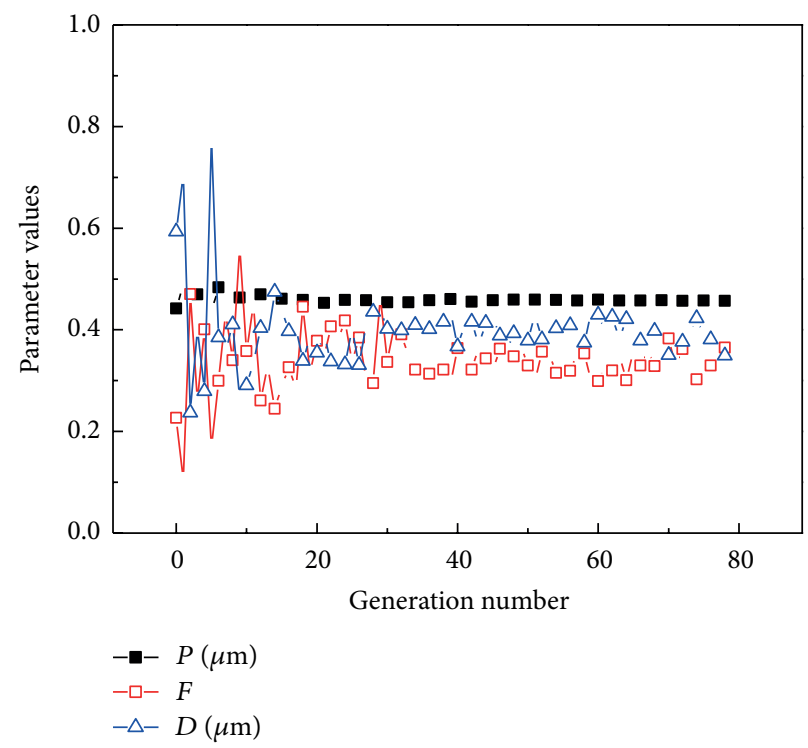

(b)

FIGURE 8: Efficiency (a) and parameter (b) convergence curves of a pure GA with respect to generation number for the TIR-based gratings with polarization-insensitive performance.

The proposed method could effectively optimize the grating structures for the purpose of polarization filter applications, and the optimized grating structures could selectively filter the incident light up to $99.8 \%$ as to TE or TM waves. The result showed its performance with high accuracy and less computation time compared to that by a single optimization technique. As a result, HTGA was confirmed as the proper optimization technique to provide the global minimum with high reproducibility, no failure, and less computation time in highly sensitive optimization problems. For the future 


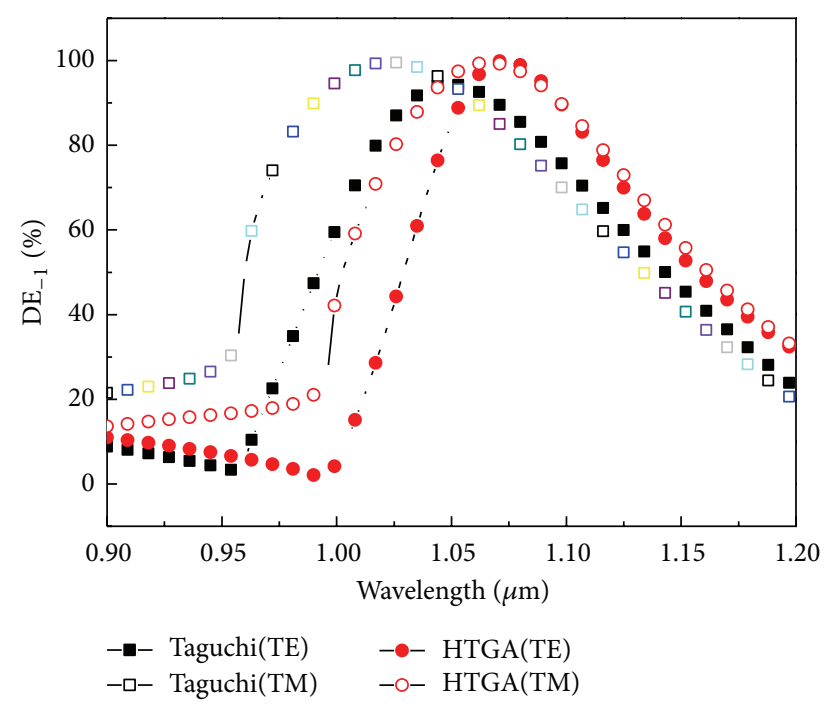

FIgURE 9: Spectral response curves on the results of the Taguchi method only $X(0.45,0.3,0.45)$ and HTGA $X(0.46,0.25,0.49)$ for the TIR-based grating with polarization-insensitive performance.

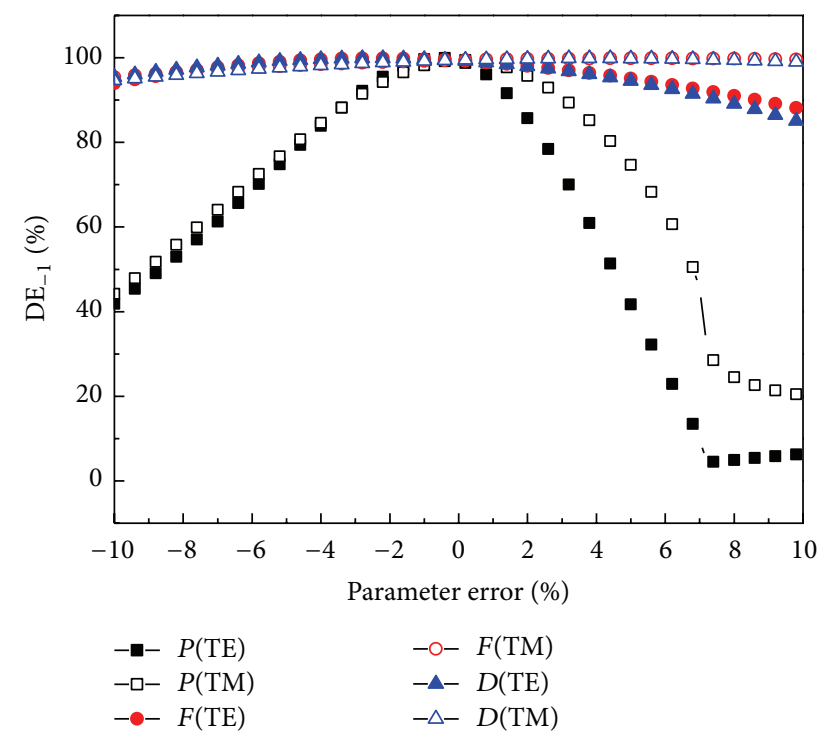

FIgURE 10: Efficiency curves with respect to parameter errors of the TIR-based gratings with polarization-insensitive performance.

work, the Taguchi method will be incorporated crossover and mutation process to select better set of parameters in a whole feasible space promptly.

\section{Conflict of Interests}

The author declares that there is no conflict of interests regarding the publication of this paper.

\section{References}

[1] M. S. D. Smith, "Diffraction gratings utilizing total internal reflection facets in littrow configuration," IEEE Photonics Technology Letters, vol. 11, no. 1, pp. 84-86, 1999.
[2] J. Song, J.-J. He, and S. He, "Fast analysis method for polarization-dependent performance of a concave diffraction grating with total-internal-reflection facets," Journal of the Optical Society of America A, vol. 22, no. 9, pp. 1947-1951, 2005.

[3] J. R. Marciante and D. H. Raguin, "High-efficiency, highdispersion diffraction gratings based on total internal reflection," Optics Letters, vol. 29, no. 6, pp. 542-544, 2004.

[4] C. Lee, K. Hane, W. Kim, and S.-K. Lee, "Design of retrodiffraction gratings for polarization-insensitive and polarizationsensitive characteristics by using the Taguchi method," Applied Optics, vol. 47, no. 18, pp. 3246-3253, 2008.

[5] F. Bahrami, M. Maisonneuve, M. Meunier, J. S. Aitchison, and M. Mojahedi, "An improved refractive index sensor based on genetic optimization of plasmon waveguide resonance," Optics Express, vol. 21, no. 18, pp. 20863-20872, 2013.

[6] S. Martim, J. Rivory, and M. Schoenauer, "Synthesis of optical multilayer systems using genetic algorithm," Applied Optics, vol. 34, no. 13, pp. 2247-2255, 1995.

[7] W. Lee and F. L. Degertekin, "Rigorous coupled-wave analysis of multilayered grating structures," Journal of Lightwave Technology, vol. 22, no. 10, pp. 2359-2363, 2004.

[8] C. Lee and S.-K. Lee, "Multi-degree-of-freedom motion error measurement in an ultraprecision machine using laser encoder-review," Journal of Mechanical Science and Technology, vol. 27, no. 1, pp. 141-152, 2013.

[9] C. Lee, K. Hane, and S. Lee, "The optimization of sawtooth gratings using RCWA and its fabrication on a slanted silicon substrate by fast atom beam etching," Journal of Micromechanics and Microengineering, vol. 18, no. 4, Article ID 045014, 2008.

[10] M. G. Moharam and T. K. Gaylord, "Rigorous coupled-wave analysis of planar-grating diffraction," Journal of the Optical Society of America, vol. 71, no. 7, pp. 811-818, 1981.

[11] M. G. Moharam, E. B. Grann, D. A. Pommet, and T. K. Gaylor, "Formulation for stable and efficient implementation of the rigorous coupled-wave analysis of binary gratings," Journal of the Optical Society of America A, vol. 12, no. 5, pp. 1068-1076, 1995.

[12] A. R. Yildiz, N. Öztürk, N. Kaya, and F. Öztürk, "Hybrid multiobjective shape design optimization using Taguchi's method and genetic algorithm," Structural and Multidisciplinary Optimization, vol. 34, no. 4, pp. 317-332, 2007.

[13] J.-T. Tsai, T.-K. Liu, and J.-H. Chou, "Hybrid Taguchi-genetic algorithm for global numerical optimization," IEEE Transactions on Evolutionary Computation, vol. 8, no. 4, pp. 365-377, 2004.

[14] D. K. Anthony, S. J. Elliott, and A. J. Keane, "Robustness of optimal design solutions to reduce vibration transmission in a lightweight 2-D structure, part I: geometric design," Journal of Sound and Vibration, vol. 229, no. 3, pp. 505-528, 2000.

[15] Y. C. Fang, T.-K. Liu, C.-M. Tsai, J.-H. Chou, H.-C. Lin, and W. T. Lin, "Extended optimization of chromatic aberrations via a hybrid Taguchi-genetic algorithm for zoom optics with a diffractive optical element," Journal of Optics A, vol. 11, no. 4, Article ID 045706, 2009.

[16] W. Y. Fowlkes and M. Clyde, Creveling, Engineering Methods for Robust Product Design, Addison-Wesley, 1995.

[17] http://www.mathworks.com.

[18] H. C. Huang, M. H. Hsu, K. L. Chen, and J. F. Huang, "Simulated annealing algorithm applied in optimum design of optical thinfilm filters," Microwave and Optical Technology Letters, vol. 38, no. 5, pp. 423-428, 2003.

[19] http://www.schott.com. 

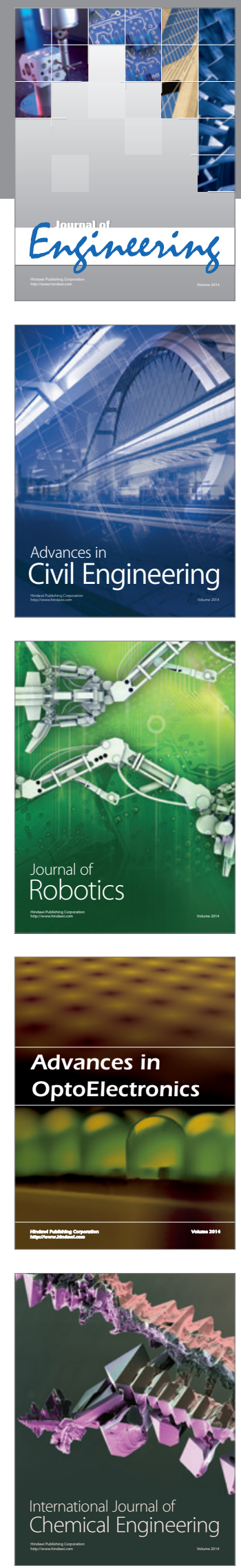

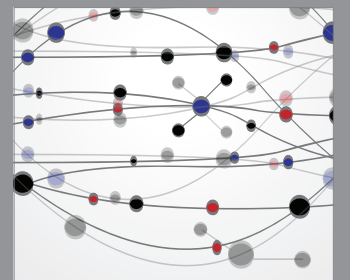

The Scientific World Journal
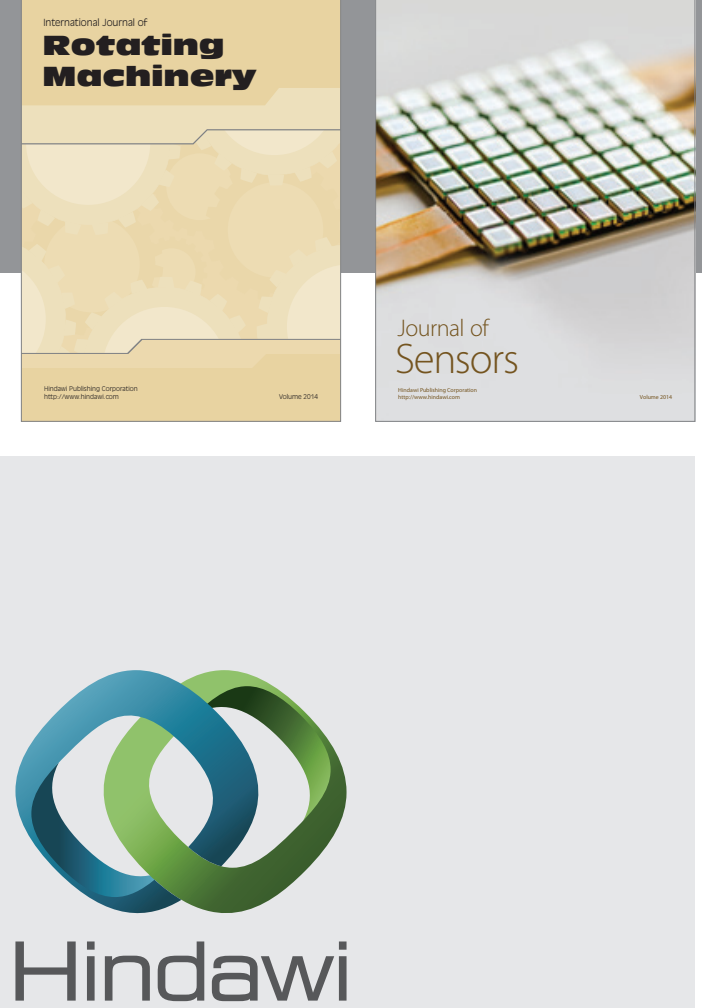

Submit your manuscripts at http://www.hindawi.com
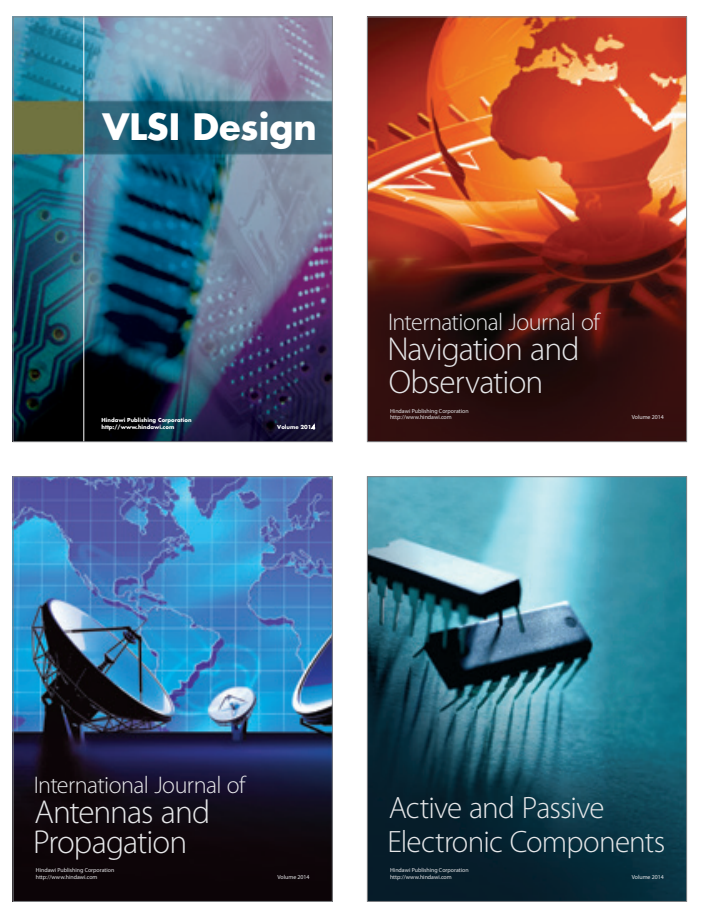
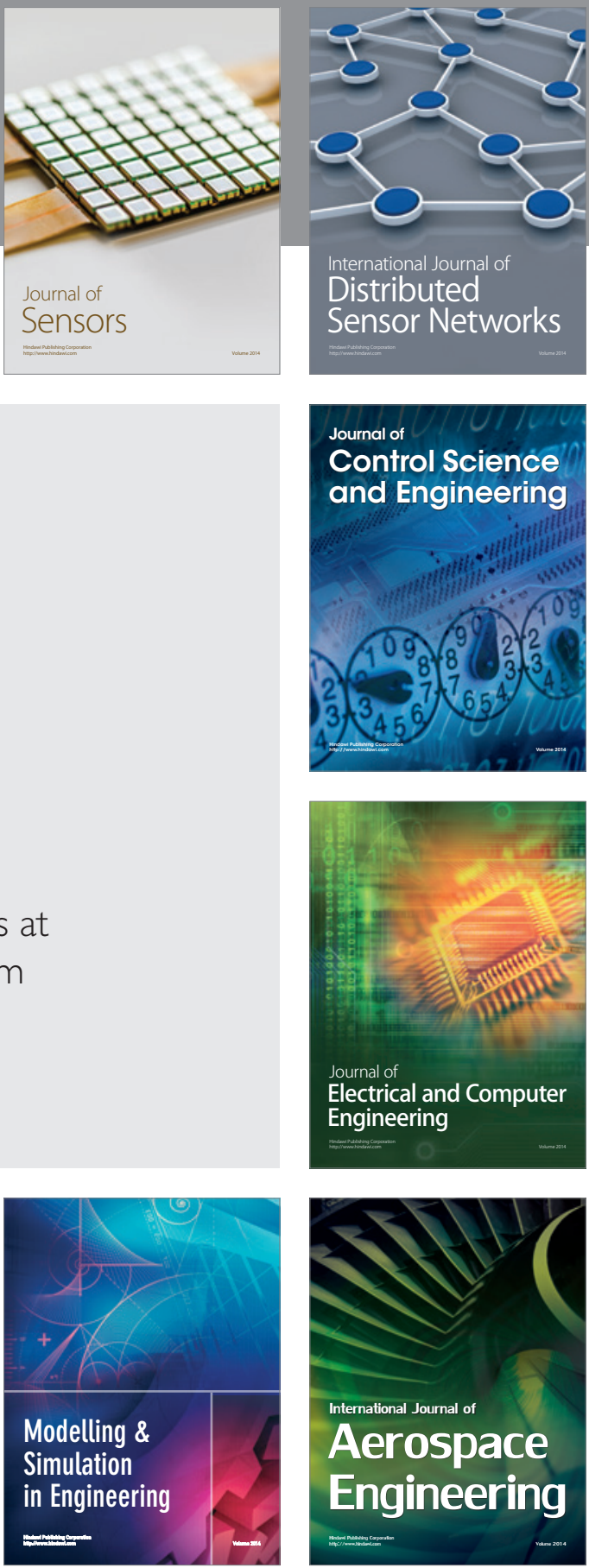

Journal of

Control Science

and Engineering
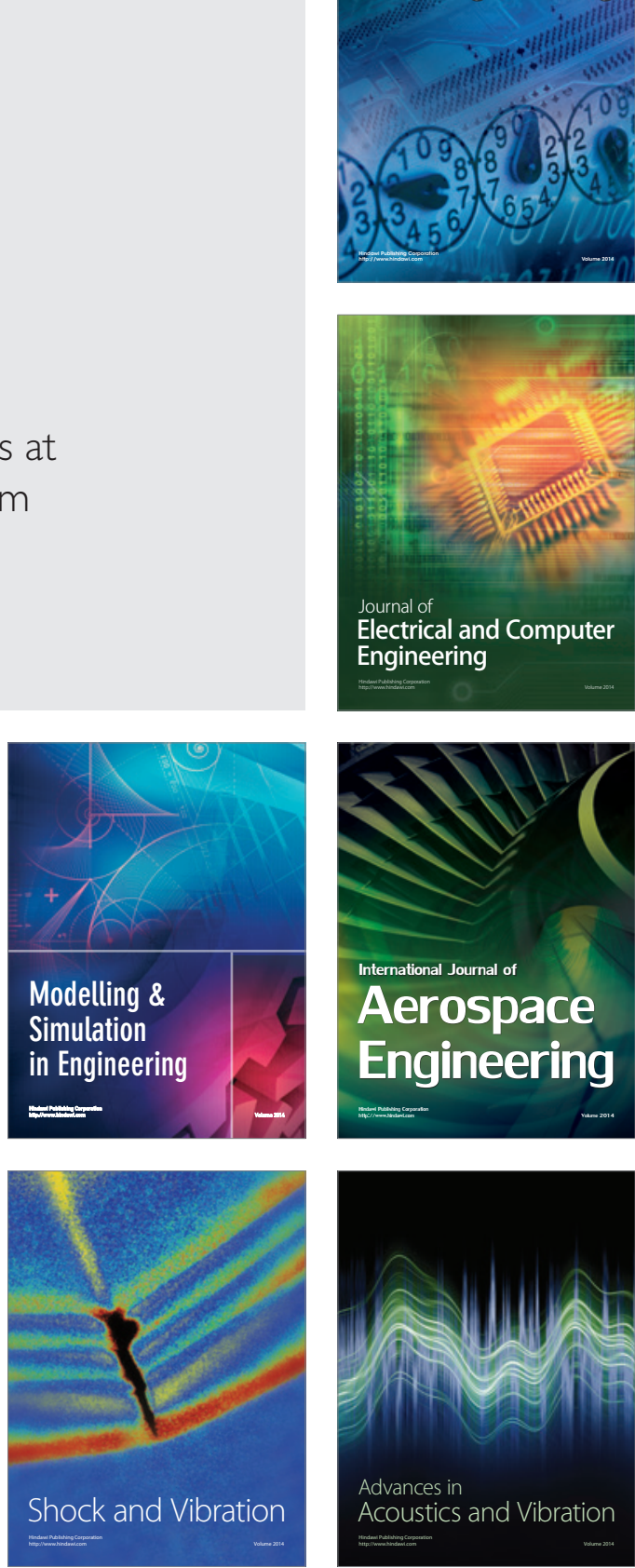